\title{
On Teaching and Training of University Students' Wind Band
}

\section{Qian Cheng}

\section{School of Music Shaanxi Normal University}

Key words: Wind band; University; University students

\begin{abstract}
Wind band of university students is an important constituent of university art organizations. It plays a critical role in developing university students' music levels, improving campus culture and strengthening school atmosphere, etc. However, in university students' wind band, there are also some teaching and training problems that need to be solved.

This paper starts from the significance of university students' wind band. It first makes a brief introduction to the current status of university students' wind band. Then, it analyzes relative problems in university students' wind band. Lastly, corresponding solutions are proposed in the hope of promoting the development of teaching and training of university students' wind band.

There are numerous instruments in university students' wind band, and most of them are Western orchestral instruments. Therefore, university students' wind band is an important constituent of artistic culture of Chinese university. To build university students' wind band can not only enrich campus culture, but also can promote the music level of university students. However, in contemporary university students' wind band, there are also some problems, which will not only inhibit the development of university students' wind band, but also will become the key to the healthy operation of university students' wind band.
\end{abstract}

\section{University students' wind band}

University students' wind band is an organization whose main performance instruments are Western orchestral instruments, and they are mainly wooden pipe, pipes and percussion instrument. The existence of university students' wind band not only plays a basic role in the construction of campus culture, but also plays an important role in training university students' music accomplishment and activating the atmosphere of campus organizations. In addition, the existence of university students' wind band is also a test standard to assess a university's comprehensive abilities. Therefore, no matter from what aspects, the existence of university students' wind band is of great importance. However, there are many problems in the teaching and training of current university students' wind band, which influence the further development and the improvement of teaching standard of university students' wind band. Based on the great importance of university students' wind band, a detailed analysis on the teaching and current training of university students' wind band is of great practical significance.

\section{Problems in the teaching and training of university students' wind band}

Firstly, from the perspective of schools, not every university has a wind band. Even if there is such a band, most of them only have intensive training when there are large-scale school activities to guarantee the standard of university students' wind band. Under such circumstances, because of insufficient attention on university students' wind band, some problems would occur in the teaching and training. 
Secondly, because of insufficient attention from the school, many university students' wind bands have very limited expenditure, and some university students' wind bands do not have enough textbooks and some even do not have textbooks. Also, some university students' wind bands do not have stable places for the teaching and practice, so the teaching and training are not stable.

Thirdly, in many universities, many university students' wind bands do not have corresponding professional teachers. Even if they have, they are not posts set for the teaching of university students' wind band. More often than not, they are chosen from music teachers temporarily for teaching and training, which leads to the result that the bands not have high-quality teachers for specific guidance and teaching. Therefore, the professional standard of university students' wind band is hard to improve, and most of them only stay at a very low level.

Fourthly, because of various reasons, there is no specific management mechanism and corresponding rewards and punishment measures for university students' wind band, which leads to the lack of sufficient cohesive force inside university students' wind band. Particularly, because of the lack of regulatory framework, many students might leave the band as they want. In the end, it leads to high mobility of personnel in university students' wind band, which is to the disadvantage of the long-term maintenance of university students' wind band. In addition, some students with excellent performance cannot acquire corresponding rewards by their excellent performance in the band, so they lose their interest in learning gradually, which results to the loss of personnel in the end.

All of the above aspects are important elements that influence the teaching and training of university students' wind band. However, the most important reason is still because many universities do not attach enough importance to university students' wind band, so it leads to the loss of textbook, expenditure and site and so on. Also, because of insufficient teaching personnel and students' low learning interest, the mobility is high inside university students' wind band.

Besides subjective elements like universities' insufficient attention on the wind band, a major difficulty for university students' wind band is also the simple professional teaching and training procedures.

Firstly, the main teaching and training way in university students' wind band is to ask students to memorize the music scores and when to play what tunes. Generally speaking, as long as students can satisfy basic performance standard, that is OK. There are not too many high standards on the performance standards of university students' wind band. Therefore, it is difficult for the teaching and training of university students' wind band to reach high standards and high requirements. In addition, in the performance standards of university students' wind band, the attention on students' basic skills is not enough. More emphasis is put on how to perform a complete piece of music with the coordination of the entire wind band in a short time. Therefore, it leads to the low performance standard of university students' wind band.

\section{Analysis on ways to promote the teaching and training of university students' wind band}

Teaching and training of university students' wind band both rely on university's resources, so universities need to make good guidance and management work.

Firstly, universities need to pay sufficient attention to the teaching and training of university students' wind band, and university students' wind band needs to play important roles in university's organizations. The important role of teaching and training in university students' wind band needs to be emphasized in various occasions, and work on thoughts also need to be considered.

Secondly, school organizations also should provide various instruments which are needed in the teaching and training of university students' wind band. In consideration of the large amount of 
different instruments and textbooks needed in the teaching and training of university students' wind band, these instruments cannot solely rely on clubs or students' personal abilities, since it would aggravate their burden and reduce their interest in learning and practicing university students' wind band. Therefore, school organizations should offer some help to the training and teaching of university students' wind band from the perspective of equipment.

Lastly, schools should also provide corresponding sites for the teaching and training of university students' wind band so that they can have fixed sites, and members can practice at ease.

Building a high-quality teacher troop is beneficial to the improvement of the standard of university students' wind band. University students' wind band does not exist as a professional team, and most of the members get together because of interests and hobbies. Therefore, this part of people have not been paid enough attention in many universities. Also, not enough attention is paid to the introduction and cultivation of high-quality teacher troop.

From this perspective, universities need to strengthen the cultivation of music teachers with high-quality teaching standard. Appropriate subsidies can be granted to teachers who teach the training of university students' wind band besides specialized courses, thereby improving these high-quality teachers' interest in building university students' wind band.

In addition, concerning the situation that there are no teachers for university students' wind band in many universities, these universities should strengthen the introduction of professional teachers for wind band to promote the mobility of talents of university students' wind band by relative talent introduction strategies and improve the standard and ability of university students' wind band.

University students' wind band does not practice and study as a professional course. Therefore, most members in the university students' wind band come from different majors with different hobbies and standards, which makes it very difficult for coordination. In order to solve this problem, a more complete management system and incentive mechanism needs to be established to promote members' interest in learning professional knowledge and training and reduce members' dropping out halfway. For example, students with excellent performance can be awarded honorary titles or awarded marks. A clear management system should be established in university students' wind band, and superiors and subordinates need to observe these plans and disciplines strictly to guarantee the effective operation of university students' wind band.

At present, music knowledge of members in university students' wind band is quite insufficient. Since they are not students of music major, their basic music knowledge need to be consolidated by professional teaching. For example, their music reading ability, music rhythm grasping ability and common terms using ability. In addition, members of university students' wind band need to have certain knowledge of music major and they need to have a detailed understanding of the creation background of common music knowledge to improve their perception ability of music.

(1) Playing posture. The quality of playing posture determines the quality of the music. Besides, it would have direct influence on the performance effects of the music scene. Also, it would influence the performing habits of members for a long time. Under such circumstances, training of basic playing skills need to conducted so that members can form a good playing habit.

(2) Breathing methods. In university students' wind band, since orchestral instruments take up a large proportion, while orchestral instruments are modulated by celiac breathing. Therefore, in teaching and training, breathing practice should be strengthened. In breathing of orchestral instruments, thoracoabdominal breathing method is usually adopted. When inhaling, the abdomen should be inflated so that space in the lung can be expanded; when expiring, certain pressure is given to the abdomen, so the result of breathing is relaxed and stable. Then, the performance of 
instruments is stable.

(3) Practice of prolonged sound and scale. Practice of prolonged sound and scale is an essential link for wind band practice. In order to guarantee the concentration of the sound, the playing of prolonged sound should be from air to sound, and the breath should be from small to large. During practice, beats should be balanced, and intonation should be controlled so that the breath is smooth and playing endurance is improved. The practice of scale is a progressive process. It should start from modes with few rise and fall marks, and it should be from shallow to deep and from slow to fast. The playing fingering is grasped during the practice of different modes.

(4) Practice of enunciation and liaison. During performance, enunciation and liaison techniques are often adopted. Enunciation is a playing method of the movement of tongues. Take the flute as an example, there are mainly playing methods such as mono-enunciation, bi-enunciation and tri-enunciation. Mono-enunciation is the basis of enunciation, and this is where beginner should start from. After grasping mono-enunciation skills, they can practice bi-enunciation. The key of the practice should be the enunciation movement at the root of tongue and the coordination of alternation movement between the tip and the root of tongue. Then, they can practice tri-enunciation. In the end, they can practice the speed of enunciation. Liaison usually suits music with strong and coherent rhythm. The purpose of the training is to have the students get used to the engagement of fingering and combine breaths with notes.

\section{Conclusion}

University students' wind band is not only an important constituent of campus culture, but also matters the presentation of a university's teaching standard and comprehensive abilities. Therefore, attention to the teaching and training of university students' wind band should be strengthened, and corresponding support should be granted so that teaching and training of university students' wind band can proceed normally, and the standard of university students' wind band can be improved practically.

\section{Bibliography:}

[1] Yu Qiaolin. Opinions on Students' Music Aesthetic Qualities from Regular Institutions of Higher Learning [J]. Home of Drama,2014(17)

[2] Huang Chao. Establishment and Training of Military Band in Regular Institutions of Higher Learning [J]. Journal of Taiyuan Urban Vocational College,2009(05)

[3] Li Yue. On the Role of Respiration in the Performance of Western Wind Instrument [D],2008.

[4] Cheng Yiming. The Art of Instrumental Ensemble of Wind Band[M]. People's Music Publishing House,2010. 